\title{
Un commentaire sur les lacunes d'une enquête de santé publique
}

\author{
John Hardie*
}

\section{Résumé}

La possibilité que l'hépatite $C$ soit transmise entre patients dentaires a été à l'origine d'une enquête rétrospective approfondie et coûteuse menée par un bureau de santé publique de l'Ontario. Cette enquête a été réalisée avec une connaissance minimale des infections nosocomiales d'origine dentaire, une confiance enthousiaste dans des indicateurs de listes de contrôle non testés et en l'absence de critères justifiant une telle enquête. Par conséquent, l'ensemble de l'exercice reposait sur la fausse prémisse qu'il y avait eu un manquement au contrôle des infections. Ce commentaire aborde ces lacunes, ainsi que d'autres aspects de la réponse du bureau de santé publique qui ont nui à sa crédibilité. Une évaluation réaliste de la transmission des maladies en dentisterie devrait être offerte afin de permettre aux bureaux de santé publique de mener des inspections éclairées et mutuellement bénéfiques des cabinets dentaires.

Citation proposée : Hardie J. Un commentaire sur les lacunes d'une enquête de santé publique. Relevé des maladies transmissibles au Canada 2021;47(11):555-8. https://doi.org/10.14745/ccdr.v47i11a10f

Mots-clés : santé publique, dentisterie, infections nosocomiales, manquements aux pratiques de prévention et de contrôle des infections
Cette oeuvre est mise à la disposition selon les termes de la licence internationale Creative Commons Attribution 4.0

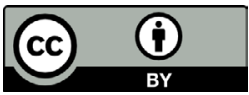

Â propos de l'auteur :

John Hardie est un pathologiste buccal à la retraite. Il a été chef de la dentisterie à l'Hôpital Civic d'Ottawa et à l'Hôpital général de Vancouver et il a occupé des fonctions similaires dans des hôpitaux et des fiducies de santé en Arabie saoudite et en Irlande du Nord. Pendant plus de 35 ans, il a donné de nombreuses conférences et publié de nombreux articles sur le contrôle des infections en ce qui concerne la dentisterie.

${ }^{*}$ Correspondance :

jhardie5@bell.net

\section{Introduction}

L'article intitulé « Intervention en santé publique à un cas nouvellement diagnostiqué d'hépatite $C$ associé à une lacune dans les pratiques de prévention et de contrôle des infections dans un établissement dentaire en Ontario, Canada » de Johnston et al. a été publié dans le bulletin de juillet/ août 2021 du Relevé des maladies transmissibles au Canada (1). Il a été rédigé du point de vue de la santé publique avec une appréciation minimale des procédures dentaires et du contrôle des infections dentaires. Par conséquent, il donne une impression biaisée de la transmission de l'hépatite $C$ en dentisterie.

En 2019, une enquête a été publiée dans le Relevé des maladies transmissibles au Canada, démontrant que le personnel des bureaux de santé publique de l'Ontario ne possède pas les connaissances, la formation et l'expertise nécessaires pour enquêter de manière appropriée sur les manquements présumés dans la prévention et le contrôle des infections dans des établissements de santé tels que les cabinets dentaires (2). Malgré ces conclusions accablantes, ces inspections se poursuivent sans relâche avec des résultats insatisfaisants pour toutes les parties concernées.

Les principales raisons sont la désinformation sur la transmission des maladies en dentisterie, la méconnaissance de la vulnérabilité des virus transmissibles par le sang et une confiance excessive dans les audits de listes de contrôle. En abordant ces sujets et d'autres sujets connexes, ce commentaire illustrera les failles de l'article ci-dessus tout en offrant une évaluation réaliste de la transmission de l'hépatite $\mathrm{C}$ dans la pratique dentaire.

\section{Transmission des maladies en dentisterie}

La présente est un résumé de la réalité de la transmission des maladies en dentisterie. 
- Selon une conclusion de 1993, « l'absence d'évidences épidémiologiques de la transmission de maladies infectieuses sur les instruments et pièces à main dentaires doit être rappelée, en particulier lors de l'évaluation d'une étude de laboratoire » (3).

- II n'y a aucun cas confirmé de transmission du virus de I'immunodéficience humaine $(\mathrm{VIH})$, du virus de l'hépatite $B$ (VHB) ou du virus de l'hépatite $\mathrm{C}(\mathrm{VHC})$ dans les cabinets dentaires canadiens (4).

- Une enquête menée sur 44 ans (1946 à 1990) dans des établissements de soins de santé (avant l'ère actuelle des recommandations sur le contrôle des infections) n'a pas permis de trouver un seul cas de contamination croisée attribuable à des instruments dentaires (5).

- Une étude approfondie réalisée en 2010 au Royaume-Uni n'a trouvé aucune preuve que les services dentaires étaient à l'origine de la transmission d'infections (6).

- Un rapport de 2013 sur la transmission du VHC dans un cabinet de chirurgie orale était basé sur de pures spéculations, comme l'ont admis les enquêteurs (7).

- Une enquête menée en 2016 aux États-Unis sur une période de 12 ans n'a pas trouvé un seul cas de transmission du $\mathrm{VIH}$ lié à un cabinet dentaire et n'a pas réussi à prouver cliniquement que les transmissions présumées du VHB et du VHC étaient dues à des manquements au contrôle des infections dentaires (8).

- En 2018, la transmission présumée d'une endocardite bactérienne dans un cabinet de chirurgie buccale était probablement liée à la préparation, au stockage et à I'utilisation inadéquats de médicaments intraveineux plutôt qu'à des lacunes présumées dans la prévention et le contrôle des infections impliquant des instruments (9).

Des années 1940 à aujourd'hui, des milliards de traitements dentaires ont été effectués, la plupart ne suivant pas les protocoles actuels de contrôle des infections. Comme il a été indiqué ci-dessus, les enquêtes historiques et actuelles n'ont pas permis de révéler que les instruments dentaires étaient des vecteurs de transmission d'infections. Dans leur hâte de mener l'enquête sur les manquements à la prévention et au contrôle des infections, les auteurs de l'article n'ont pas effectué un examen approfondi de la littérature pertinente.

\section{Vulnérabilité des virus transmissibles par le sang}

Selon l'Agence ontarienne de protection et de promotion de la santé : " la désinfection de faible niveau élimine les bactéries dans leurs formes végétatives (vivantes), certains champignons et les virus enveloppés » (10). Le virus de l'hépatite $C$ est un virus à enveloppe lipidique qui est facilement détruit par les désinfectants courants utilisés par des générations de dentistes. Les auteurs de l'article croient-ils que le VHC survivrait au nettoyage physique, à l'immersion dans un désinfectant de faible niveau et à l'environnement difficile d'un stérilisateur à vapeur, même s'il fonctionnait avec une efficacité inférieure à $100 \%$ ?

Le VIH et le VHB sont également des virus à enveloppe lipidique. Ce fait, associé à la faible pathogénicité des micro-organismes buccaux, explique l'absence de preuves cliniquement fondées que les instruments dentaires transmettent des infections. Il est regrettable que les responsables de la santé publique continuent d'ignorer ces données.

\section{Dépendance aux audits de liste de contrôle}

Les listes de contrôle de Santé publique Ontario ont été utilisées pour déterminer s'il y a eu des manquements à la prévention et au contrôle des infections. Ces listes contiennent environ 100 indicateurs dont l'adoption est censée prévenir ou contrôler les infections acquises par voie dentaire. L'Agence ontarienne de protection et de promotion de la santé exige que ces indicateurs soient « ...fondés sur des preuves validées dont il a été démontré qu'elles améliorent les résultats » (9). Autrement dit, il doit y avoir des preuves cliniques de la transmission d'une maladie avant l'utilisation de l'indicateur, qui a été prévenue ou contrôlée après l'adoption de l'indicateur.

L'article cite 14 indicateurs déficients pour justifier la présence de manquements à la prévention et au contrôle des infections. Il s'agit notamment de l'utilisation incohérente des indicateurs chimiques, de la tenue incomplète des registres et du nettoyage inadéquat des pièces à main dentaires. Cependant, aucune étude clinique ne démontre que ces manquements et les autres manquements dans les listes de contrôle sont à l'origine $d$ 'infections nosocomiales d'origine dentaire qui auraient été évitées si l'on respectait les indicateurs. La réalité est que les listes de contrôle n'ont pas été validées. Cela ne devrait pas être surprenant; Nicolle a noté dans le Journal canadien des maladies infectieuses que « les interventions de lutte contre les infections n'ont pas encore été validées dans les établissements de santé en dehors des soins aigus» (11).

Sans la validation exigée par l'Agence ontarienne de protection et de promotion de la santé, il n'est pas possible de déterminer les lacunes dans la prévention et le contrôle des infections, et sans cette détermination, il est inapproprié et malhonnête de suggérer dans le titre de l'article qu'il en existe une. 


\section{Sujets connexes}

Les points suivants nuisent davantage à la crédibilité de l'article :

- Caractère unique du génotype 2 de I'hépatite $C:$ Le taux déclaré d'hépatite $C$ en Ontario est de 36,5 pour 100000 , dont $10 \%$ à $15 \%$ sont de génotype 2 (12). Sur une population de 15 millions, il y aura environ 550 à 800 cas d'hépatite de génotype 2. Cela contredit les affirmations des auteurs selon lesquelles la rareté du génotype 2 confère un caractère unique à son article.

- Catégories de risques : Le statut socio-économique des patients concernés n'est pas connu. Cependant, ils ont été traités dans des cliniques dentaires communautaires, ce qui met en doute leur fiabilité en tant qu'historiens de leur santé, de leurs activités sexuelles et récréatives nécessitant une enquête approfondie sur leurs facteurs de risque pour I'hépatite $C$. Ceci n'est pas mentionné dans l'article. Au lieu de cela, il y a une référence passagère au fait que le patient index n'avait aucun « ...facteur de risque actuel ou passé signalé lié à l'infection par le VHC » (1).

- Enquête rétrospective : L'article décrit une enquête rétrospective. Le Dr Danila et son équipe l'ont classée comme étant coûteuse et limitée dans sa capacité à démontrer la transmission en raison du nombre relativement faible de patients examinés et d'un faible risque de transmission (13). Cette enquête a nécessité 1187,5 heures, ce qui pourrait se traduire par une abondance d'argent du contribuable. Les auteurs admettent qu'il existe « ...peu de preuves scientifiques de la transmission du VHC dans la pratique dentaire ». Cet aveu aurait dû suffire à semer le doute quant à la réussite de l'enquête. Trois critères justifient les enquêtes rétrospectives (13) : 1) la preuve formelle de la transmission de la maladie à un patient; 2) des violations flagrantes du contrôle des infections; et 3) le cadre d'une étude collaborative. Cette enquête ne satisfait à aucun de ces critères.

- Erreurs éditoriales : Dans la section « Définition du cas", les jours précédant et suivant la procédure sont classés comme des jours « ouvrables ». Cependant, dans la section "Discussion », le terme « entreprise » est absent. Dans la section "Résultats", le cas source est décrit comme "le cas source probable ». Dans la section «Discussion », ce point s'est transformé en " cas source " définitif sans aucune justification rationnelle.

- Hypothèse impossible : Dans l'introduction, l'épidémie de VHC est déclassée en épidémie " potentielle » et les expositions au VHC sont considérées comme des expositions « potentielles ». Cela signifie que I'hypothèse des auteurs devrait se lire comme suit : « ... II peut y avoir une transmission potentielle dans l'établissement $B$ entre le cas source probable et le cas index ". Les corrections en gras sont telles qu'il serait impossible de tester l'hypothèse, ce qui la rend invalide.

\section{Dentisterie et l'hépatite C}

Les endoscopes sont fortement contaminés pendant leur utilisation et leur conception complexe entraîne des erreurs de retraitement. La conception des instruments dentaires est plus simple; ils ne sont pas soumis à la même charge biologique et sont souvent utilisés pour des procédures non invasives. Les enquêtes sur le risque de transmission d'infections transmissibles par le sang par des endoscopes oto-rhino laryngologiques retraités de manière inadéquate servent de scénarios catastrophes de manquements au contrôle et à la prévention des infections lors de la décontamination des instruments dentaires. De telles études ont montré que le risque de transmettre le $\mathrm{VIH}$ est de 7 sur 10 billions, pour le VHB il est de 2,4 sur un milliard et pour le VHC il se situe entre celui du VIH et du VHB (13). Ces résultats prouvent qu'il existe un risque infinitésimal de contracter des infections transmissibles par le sang, notamment I'hépatite $C$, à partir d'instruments dentaires. Les auteurs semblent ne pas avoir connaissance de ces évaluations des risques.

\section{Conclusion}

La réponse de la santé publique était un exercice administratif, car elle n'était pas basée sur une épidémie réelle de VHC, mais sur une épidémie potentielle. Bien qu'elle ait nécessité plus d'un millier d'heures de travail de la part du personnel, elle était imparfaite en ce sens qu'elle s'appuyait sur des listes de contrôle non validées, qu'il n'y avait aucune justification clinique pour mener l'enquête rétrospective, qu'elle exagérait le caractère unique du cas et qu'elle était fondée sur une hypothèse qui ne peut être testée. Les pairs des auteurs apprécieront la valeur de la réponse à un manquement à la prévention et au contrôle des infections non prouvées.

Comme expliqué ci-dessus, il existe des raisons historiques et factuelles expliquant la rareté des transmissions de maladies cliniquement prouvées par les instruments dentaires. Peut-être les responsables de la santé publique utiliseront ces raisons pour prendre en compte la réalité de la transmission des maladies en dentisterie, ce qui leur permettra de mener des enquêtes plus éclairées sur les pratiques dentaires à l'avenir.

\section{Références}

1. Johnston C, Sunil V, Ser D, Holt AM, Garber G, Macdonald L, Kristjanson E, Mazzulli T, Olsha R, Ryding D, Noseworthy AL. Intervention en santé publique à un cas nouvellement diagnostiqué d'hépatite $C$ associé à une lacune dans les pratiques de prévention et de contrôle des infections dans un établissement dentaire en Ontario, Canada. Relevé des maladies transmissibles au Canada 2021;47(7/8):382-8. DOI 
2. Cadieux G, Brown C, Sachdeva H. Enquête des plaintes relatives aux pratiques de prévention et de contrôle des infections par la santé publique en Ontario, de 2015 à 2018. Relevé des maladies transmissibles au Canada 2019;45(11):318-25. DOI

3. Epstein JB, Rea G, Sibau L, Sherlock CH. Rotary dental instruments and the potential risk of transmission of infection: herpes simplex virus. J Am Dent Assoc 1993;124(12):55-9. DOI PubMed

4. Hardie J. The Surprising Absence of Disease Transmission from Infection Control Disasters. Oral Health 2015;14-8. https://www.oralhealthgroup.com/features/the-surprisingabsence-of-disease-transmission-from-infection-controldisasters/

5. Goodman RA, Solomon SL. Transmission of infectious diseases in outpatient health care settings. JAMA 1991;265(18):2377-81. DOI PubMed

6. Fox C. Evidence summary: what 'cost of illness' evidence is there about cross-infection related infections in dental practice? Br Dent J 2010;209(2):87-8. DOI PubMed

7. Weaver JM. Confirmed transmission of hepatitis $C$ in an oral surgery office. Anesth Prog 2014;61(3):93-4. DOI PubMed

8. Cleveland JL, Gray SK, Harte JA, Robison VA, Moorman AC, Gooch BF. Transmission of blood-borne pathogens in US dental health care settings: 2016 update. J Am Dent Assoc 2016;147(9):729-38. DOl PubMed

9. Ross KM, Mehr JS, Greeley RD, Montoya LA, Kulkarni PA, Frontin S, Weigle TJ, Giles H, Montana BE. Outbreak of bacterial endocarditis associated with an oral surgery practice: new Jersey public health surveillance, 2013 to 2014. J Am Dent Assoc 2018;149(3):191-201. DOI PubMed
10. Public Health Ontario. Provincial Infectious Diseases Advisory Committee (PIDAC). Best Practices for Infection Prevention and Control Programs in Ontario. In All health Care Settings, $3^{\text {rd }}$ edition. Toronto, ON: PHO; (modifié 2012-05). https://www.publichealthontario.ca/-/media/ documents/B/2012/bp-ipac-hc-settings.pdf

11. Nicolle LE. Infection control in acute care facilities: evidence-based patient safety. Can J Infect Dis 2001;12(3):131-2. DOl PubMed

12. Public Health Ontario. Hepatitis C in Ontario, 2018: Surveillance summary one year after a case definition update. Toronto (ON): PHO; 2020 (accédé 2020-09-30). https://www.publichealthontario.ca/-/media/ documents/r/2020/report-hepc-surveillance-2018.pdf?la=en

13. Danila RN, MacDonald KL, Rhame FS, Moen ME, Reier DO, LeTourneau JC, Sheehan MK, Armstrong J, Bender ME, Osterholm MT. A look-back investigation of patients of an HIV-infected physician. Public health implications. N Engl J Med 1991;325(20):1406-11. DOI PubMed

14. Holodniy M, Oda G, Schirmer PL, Lucero CA, Khudyakov YE, Xia G, Lin Y, Valdiserri R, Duncan WE, Davey VJ, Cross GM. Results from a large-scale epidemiologic look-back investigation of improperly reprocessed endoscopy equipment. Infect Control Hosp Epidemiol 2012;33(7):649-56. DOI PubMed 